\title{
Can Serial Measurement Enhance the Diagnostic Value of Procalcitonin as a Marker of Gram-negative Bacteremia in Children with Acute Leukemia?
}

\author{
(1) Zümrüt Şahbudak Bal1', (1) Gülinaz Ercan², (1) Deniz Yılmaz Karapınar³ \\ ${ }^{1}$ Ege University Faculty of Medicine, Department of Pediatrics, Division of Infectious Diseases, İzmir, Turkey \\ 2Ege University Faculty of Medicine, Department of Medical Biochemistry, İzmir, Turkey \\ ${ }^{3}$ Ege University Faculty of Medicine, Department of Pediatrics, Division of Hematology, İzmir, Turkey
}

\begin{abstract}
Aim: Despite improvements in diagnosis and treatment, infections are still major cause of morbidity and mortality in children with febrile neutropenia (FN). In these patients, due to inadequate inflammatory response and subtle clinical symptoms, to determine the source of infection can be challenging. Therefore, it is important to distinguish infections from other non-infectious causes, for both to choose appropriate antibiotic and to reduce the redundant antibiotic use.

Materials and Methods: In this retrospective study, we aim to evaluate serial procalcitonin (PCT) levels for predicting bacteremia particularly caused by Gram-negative microorganism.

Results: Among FN episodes caused by Gram-negative microorganism, the median level of second PCT sample obtained between 48 and 72 hours PCT 2 was found to be significantly higher when compared to infections caused by Coagulase-negative Staphylococcus or culturenegative confirmed infections, p value was 0.003; however, fever onset PCT 1 and C-reactive protein (CRP) 1 values showed no significant difference $(p>0.05)$. The area under curve values demonstrated by receiver operating characteristic (ROC) analysis for CRP 1, CRP 2, PCT 1, PCT 2 were $0.664,0.748,0.504$ and 0.842 , respectively.

Conclusion: This study showed that initial PCT levels were not significantly correlate with culture-confirmed bacterial infection. Therefore, initial PCT values do not help the clinicians in terms of administering or postponing empirical antibiotics at the time of fever onset. However, third day PCT levels present as a good diagnostic marker due to a higher sensitivity and specificity when comparing them to the initial values. Determination of serial PCT may enhance the diagnostic value of PCT diagnostic marker in FN episodes caused by Gram-negative bacteria with a high sensitivity (87.5\%). This study also demonstrated that PCT could be used to rule out bacterial infections particularly caused by Gram-negative bacteria.
\end{abstract}

Keywords: Procalcitonin, febrile neutropenia, child, Gram-negative bacteremia 


\section{Introduction}

Infections are the most prominent cause of morbidity and mortality in children with febrile neutropenia (FN) $(1,2)$. In these patients, due to an inadequate inflammatory response and subtle clinical symptoms, determining the source of infection can be challenging. Fever sometimes presents as the primary and sole manifestation of an infection adding to the confusion. A positive microbiological culture is only found in 7-31\% of febrile episodes (3). Therefore, it is important to distinguish infections from other non-infectious causes, both to choose an appropriate antibiotic and to reduce redundant antibiotic use. A focus of recent studies has been a search for predictors of severe infection and bacteremia. Procalcitonin (PCT) is a precursor of the calcitonin hormone and is excreted in the early phase of infections $(4,5)$. There are several studies suggesting PCT as a better and earlier marker than C-reactive protein (CRP) in children with infections (6-9). A recent meta-analysis evaluating 10 pediatric studies has suggested that PCT is a useful biomarker in detecting microbiologically/clinically confirmed infections with a sensitivity and specificity of $68 \%$ and $82 \%$, respectively (6). Another pediatric study has also shown that PCT $>2$ $\mathrm{ng} / \mathrm{dL}$ was a good predictor of severe infection [likelihood ratio (LHR) of 26 (95\% confidence interval (CI) 3.5, 190)] (9).

This study was conducted to investigate the possible use of PCT levels being used for predicting bacteremia particularly those caused by Gram-negative microorganisms; which are respectively more frequent in our center. The second aim of this study was to determine whether serial measurements of PCT could improve diagnostic value.

\section{Materials and Methods}

Thirty-three patients with 51 febrile neutropenia episodes who were hospitalized in the Pediatric Hematology Subdivision of Ege University between February 2014 and June 2014 were evaluated. The patients aged between 1 month and 18 years old were receiving a chemotherapy regimen for acute lymphoblastic leukemia (ALL) $(n=27)$ or acute myeloblastic leukemia (AML) $(n=6)$. During this period, $51 \mathrm{FN}$ episodes were observed. The measurements of CRP 1 and PCT 1 were performed at onset of fever (0-24 hour) and re-measurement was performed within 48-72 hour (CRP 2, PCT 2). Blood cultures were obtained at the onset of fever before the initiation of antibiotics. All patients already receiving antibiotic therapy were excluded. Febrile neutropenia was defined according to the IDSA guideline (10). Bacteremia was defined in patients with a positive blood culture for bacteria (peripheral blood or central venous indwelling catheter), with or without shock. Standard practice in our center includes a thorough daily examination of all patients for clinical signs and sources of infections, as well as monitoring for sepsis and septic shock. Initial blood samples are obtained on the first day and the second draw being performed on the third day at the time the reassessment of antibiotic therapy is recommended. Broad spectrum antibiotics (piperacillintazobactam+amikacin or meropenem+amikacin) are initiated after performing blood cultures. Additionally, when an infection is suspected, a urine culture and a cerebrospinal fluid culture are obtained. In those patients with hemodynamic instability, skin and soft tissue infections, or signs of catheter-related infection, vancomycin is initiated empirically.

The medical records of 33 patients whose initial PCT and CRP levels were available were evaluated. The CRP and PCT levels of these patients were also checked and recorded if they were drawn on the third day of admission. The demographic characteristics, medical history, maximum degrees of fever on the day of presentation, fever duration and physical examination findings were recorded. Laboratory findings, including complete blood count, CRP, bacterial cultures and fungal cultures were also recorded. This study was approved by the Ethics Board of Ege University (approval number: 13-4.1/12). All of the parents of the patients gave their informed consent prior to their child's inclusion in the study.

\section{Microbiological Testing}

The identification of microorganism was made using VITEK MS (bioMérieux, France). This technology uses Matrix assisted laser desorption ionization time of flight mass spectrometry, which is a new technology used for the identification of species based on the protein composition of microbial cells.

\section{Statistical Analysis}

Statistical analyses were performed using MedCalc for Windows (version 15.2 MedCalc Software, Belgium) and SPSS for Windows (version 22.0 SPSS Inc., Chicago, IL, USA). Numerical data were expressed as median (25P-75P). Mann-Whitney $U$ test was used for inter-variable analysis. Comparisons were referred to as statistically significant if the $p$ values were $<0.05$.

\section{Results}

This retrospective study consisted of 51 febrile episodes from 33 patients $\operatorname{ALL}(n=27)$ and AML $(n=6)$ with a median age of 92.1 months ranging between 13 and 216 months. The initial PCT levels of 51 episodes, and third day PCT levels of 41 episodes were available (Table I). The mean durations of fever and neutropenia were 3.82 (1-19) days and 9.33 (0-56) days, respectively. Five (9.8\%) patients developed septic shock with $4(7.8 \%)$ of them requiring intensive care unit (ICU) admission. The majority of FN episodes [13 (25.4\%)] were associated with catheter related bloodstream infection (BSI). This was followed by pneumonia: 3 episodes 
(5.8\%), catheter exit site infection: 1 episode (1.9\%) and zona zoster infection: 1 episode (1.9\%). From 13 (25.4\%) catheter-related BSI episodes, blood cultures were positive for Gram-positive microorganism in 5 episodes and Gramnegative microorganism in 8 episodes. In all culture-

Table I. Demographic and clinical characteristics of febrile neutropenia episodes

\section{Characteristics of episodes}

\begin{tabular}{|l|l|}
\hline Total number of episodes, $\mathrm{n}(\%)$ & $51(100)$ \\
\hline $\begin{array}{l}\text { Duration of fever, median } \\
\text { (minimum-maximum) (days) }\end{array}$ & $3.82(1-19)$ \\
\hline $\begin{array}{l}\text { Duration of neutropenia, median } \\
\text { (minimum-maximum) (days) }\end{array}$ & $9.33(0-56)$ \\
\hline Intensive care unit admission, $\mathrm{n}(\%)$ & $4(7.8)$ \\
\hline Septic shock, $\mathrm{n}(\%)$ & $5(9.8)$ \\
\hline Culture-confirmed infections, $\mathrm{n}(\%)$ & $13(25.4)$ \\
\hline Coagulase-negative Staphylococcus, $\mathrm{n}(\%)$ & $5(9.8)$ \\
\hline Klebsiella pneumoniae, $\mathrm{n}(\%)$ & $3(5.8)$ \\
\hline Escherichia coli, $\mathrm{n}(\%)$ & $3(5.8)$ \\
\hline Pseudomonas aeruginosa, $\mathrm{n}(\%)$ & $2(3.9)$ \\
\hline
\end{tabular}

confirmed episodes, the median levels of CRP and PCT on the first day, and on the third day were not found to be statistically different with $p$ levels of 0.494, 0.755, 0.326, 0.592, respectively. However, in Gram-negative microorganism caused infections, the median levels of PCT 2 and CRP 2 showed significantly higher levels when compared to infections caused by CoNS or culture-negative confirmed infections, $p$ values were 0.029 and 0.003 , respectively. PCT 1, CRP 1 were not statistically different $(p>0.05)$ (Table II).

In the Gram-negative bacteria infected group and the non-infected group, the values of sensitivity, specificity, positive LHR, negative LHR for CRP 1, CRP 2, PCT 1, PCT 2 demonstrated using ROC curves, are given in Table III. The area under the curve (AUC) showed a statistical significance for CRP 2 and PCT 2 levels $(p=0.0015$ and $p<0.0001$, respectively). However, CRP 1 and PCT 1 levels showed no significance ( $p>0.05)$. The AUC values for CRP 1, CRP 2, PCT 1, and PCT 2 were $0.664,0.748,0.504$ and 0.842 , respectively. This is summarized in Table III. The optimal cut off values, calculated using ROC curve analysis, were found to be $4 \mathrm{mg} /$ $\mathrm{dL}$ for CRP1, $1.3 \mathrm{mg} / \mathrm{dL}$ for CRP 2, $0.29 \mu \mathrm{g} / \mathrm{L}$ for PCT 1 and 0.17 $\mu / L$ for PCT 2.

Table II. Comparison of CRP 1, CRP 2, PCT 1, PCT 2 values in febrile neutropenic patients infected with Gram-negative bacteria and noninfected

\begin{tabular}{|l|l|l|l|}
\hline & $\begin{array}{l}\text { Gram-negative bacteria infected } \\
{[\mathbf{n = 8}(\mathbf{1 5 . 7 \%})]}\end{array}$ & $\begin{array}{l}\text { Non-Gram-negative bacteria infected } \\
{[\mathbf{n}=\mathbf{4 3}(\mathbf{8 4 . 3 \% )}]}\end{array}$ & $\mathbf{p}$ value \\
\hline CRP 1, median (IQR) & $2.80(3.5)$ & $4.40(5.43)$ & 0.152 \\
\hline CRP 2, median (IQR) & $2.60(1.1)$ & $0.795(2.53)$ & 0.029 \\
\hline PCT 1, median (IQR) & $0.31(0.41)$ & $0.295(0.297)$ & 1.000 \\
\hline PCT 2, median (IQR) & $0.40(4.64)$ & $0.155(0.1)$ & 0.003 \\
\hline
\end{tabular}

CRP 1 (Fever onset): C-reactive protein, PCT 1 (Fever onset): Procalcitonin, CRP 2 (48-72 hour), PCT 2 (48-72 hour), IQR: Interquartile range

Table III. Diagnostic accuracy of PCT and CRP for predicting Gram-negative bacteremia, results from receiver operating curve analysis

\begin{tabular}{|l|l|l|l|l|}
\hline & CRP 1 (FO) & CRP 2 (48-72 hr) & PCT 1 (FO) & PCT 2 (48-72 hr) \\
\hline Cut-off value & $4 \mathrm{mg} / \mathrm{dL}$ & $1.3 \mathrm{mg} / \mathrm{dL}$ & $0.50 \mu / \mathrm{dL}$ & $0.17 \mu \mathrm{g} / \mathrm{dL}$ \\
\hline Sensitivity (\%) & 87.5 & 87.5 & 37.5 & 85.7 \\
\hline Specificity (\%) & 47.6 & 66.7 & 79.0 & 69.7 \\
\hline PPV (\%) & 22.6 & 38.9 & 25 & 37.5 \\
\hline NPV (\%) & 95.2 & 95.7 & 87.2 & 95.8 \\
\hline Positive LHR (95\% Cl) & $1.67(1.1-2.5)$ & $2.62(1.5-4.5)$ & $1.79(0.6-5.2)$ & $2.83(1.6-5.2)$ \\
\hline Negative LHR (95\% Cl) & $0.26(0.04-1.7)$ & $0.19(0.03-1.2)$ & $0.79(0.5-1.4)$ & $0.20(0.03-1.3)$ \\
\hline AUC (95\% Cl) & $0.664(0.516-0.791)$ & $0.748(0.588-0.870)$ & $0.504(0.361-0.647)$ & $0.842(0.692-0.938)$ \\
\hline SE & 0.122 & 0.0763 & 0.119 & 0.0705 \\
\hline P value & 0.1793 & 0.0011 & 0.9736 & $<0.0001$ \\
\hline
\end{tabular}

PCT: Procalcitonin, CRP: C-reactive protein, FO: Fever onset, PPV: Positive predictive value, NPV: Negative predictive value, LHR: Likelihood ratio, Cl: Confidence interval, AUC: Area under curve, SE: Standard error 


\section{Discussion}

Children with chemotherapy induced neutropenia may develop infections which rapidly progress to sepsis. Fever can be an initial and the sole manifestation of these infections. Therefore, patients presenting with fever require the initiation of broad-spectrum antibiotics immediately. Using reliable bacterial infection markers can allow health care providers to make an accurate clinical decision regarding antibiotic use and additionally the unnecessary use of antibiotics can be avoided. PCT has been shown to be a reliable marker for distinguishing from other noninfectious causes in patients with FN (7).

CRP and PCT are the biomarkers most commonly preferred in clinical use for predicting bacteremia. In this retrospective study, PCT levels in the second draw on the third day were found to have better diagnostic accuracy than initial values; particularly in detecting FN episodes caused by Gram-negative microorganisms. Gramnegative bacterial infections were satistically significantly associated with higher levels of CRP and PCT on the $3^{\text {rd }}$ day of FN. Both CRP and PCT levels were found to be valuable markers due to their high negative predictive value (NPVs). However, the positive predictive value were found to be low. In a recent study comparing PCT levels in pediatric patients with $\mathrm{FN}$ against healthy controls, PCT levels of patients with FN were significantly higher than the controls' $(p=0.001)$. However, the cultureconfirmed infections and fever of unknown origin were not compared (11). The malign process itself and also results such as mucositis and graft versus host disease have been shown to stimulate chemokines and to be associated with increased inflammatory markers including CRP, erythrocyte sedimentation rate and leukocyte count. Therefore, investigations focused on PCT as a promising diagnostic marker. A recent meta-analysis evaluated 3.420 FN episodes and the lowest area under curve was found in immunocompromised patients when compared with non-immunocompromised patients and ICU patients (4). Demirkaya et al. (12) evaluated 50 FN episodes of 37 cancer patients and found that PCT levels were seen to be higher in patients with sepsis than those clinically and microbiologically documented infections at admission and on day 3 and day 7. In our study, PCT did not show a significant difference at day of fever onset while its diagnostic value had improved on day 3 .

A prospective cohort study by Hemming et al. (9), which included 27 patients with 48 FN episodes, demonstrated that PCT $>2 \mathrm{~g} / \mathrm{dL}$ was strongly associated with an increased risk of severe infection [LHR of 26 (95\% CI: 3.5, 190). Several previous reports evaluating the diagnostic value of PCT have reported sensitivity and specificity ranging between 93\%-96.5\% and $70.6 \%-97 \%$, respectively, in children with fever induced due to chemotherapy (13-15). In contrast, another large prospective cohort evaluated 194 consecutive FN episodes and found that fever onset median PCT levels did not differ between infections and fevers of unexplained origin. However, the diagnostic value did increase on the second day of fever (56\% sensitivity, $90 \%$ specificity) which is similar to our findings (16). Stoma et al. (17) suggested that $\mathrm{PCT}$ is a good diagnostic marker with a $62 \%$ sensitivity and an $88 \%$ specificity in adult patients with Gram-negative BSI following hematopoietic stem cell transplant. In our study, at fever onset, PCT levels showed a lower specificity (51.1\%) in predicting BSI caused by Gram-negative microorganisms. The lower specificity could be attributed to the low ratio of documented infections. On the other hand, higher PCT levels were significantly associated with Gram-negative bacteremia ( $p=0.018$ ) on the third day. Fleischhack et al. (18) determined that the PCT levels were significantly higher in children with febrile neutropenia caused by a Gram-negative bacteria. Similarly, Reitman et al. (19) reported higher levels of PCT in febrile neutropenic children infected with Gramnegative bacteria than those infected with Gram-positive bacteria. In their study, PCT levels on admission showed a sensitivity of $50 \%$ and a specificity of $79 \%$. However, a serial analysis of PCT showed a sensitivity of $78 \%$ and a specificity of $76 \%$ with an NPV of $96 \%$. They also suggested PCT as a good biomarker for ruling out bacteremia due to high NPV. In this study, we also found third day PCT levels showed an NPV of $95.8 \%$ which is similar to previous reports.

The limitations of this study included a relatively low number of $\mathrm{FN}$ episodes and its retrospective design.

\section{Conclusion}

This study shows that initial PCT levels were not significantly corelated with culture-confirmed bacterial infection. Therefore, initial PCT values do not help clinicians in terms of administering or postponing empirical antibiotics at the time of fever onset. However, third day PCT levels are a good diagnostic marker due to a higher sensitivity and specificity when compared to initial values. This study also demonstrated that PCT could be used to rule out bacterial infections particularly those caused by Gram-negative bacteria. Due to a low rate of culture-confirmed infections in children with chemotherapy-induced neutropenia, monitoring PCT may provide a platform by which antibiotic therapy can be more accurately managed.

\section{Ethics}

Ethics Committee Approval: This study was approved by Ethics Board of Ege University (approval number: 13-4.1/12).

Informed Consent: All of the parents of the patients gave their informed consent prior to their child's inclusion in the study.

Peer-review: Externally peer-reviewed. 


\section{Authorship Contributions}

Concept: Z.Ş.B., Design: Z.Ş.B., Data Collection or Processing: Z.Ş.B., G.E., Analysis or Interpretation: Z.Ş.B., Literature Search: Z.Ş.B., Writing: Z.Ş.B., D.Y.K.

Conflict of Interest: None of the authors had conflict of interest.

Financial Disclosure: The authors declared that this study received no financial support.

\section{References}

1. Barton CD, Waugh LK, Nielsen MJ, Paulus S. Febrile neutropenia in children treated for malignancy. J Infect 2015;71(Suppl 1):27-35.

2. Haeusler GM, Sung L, Ammann RA, Phillips B. Management of fever and neutropenia in paediatric cancer patients: room for improvement? Curr Opin Infect Dis 2015;28:532-8.

3. de Naurois I, Novitzky-Basso I, Gill M), et al. Management of febrile neutropenia: ESMO Clinical Practice Guidelines. Ann Oncol 2010;2:252-6.

4. Hoeboer $\mathrm{SH}$, van der Geest PJ, Nieboer D, Groeneveld AB. The diagnostic accuracy of procalcitonin for bacteraemia: a systematic review and meta-analysis. Clin Microbiol Infect 2015;21:474-81.

5. Wacker C, Prkno A, Brunkhorst FM, Schlattmann P. Procalcitonin as a diagnostic marker for sepsis: a systematic review and metaanalysis. Lancet Infect Dis 2013;13:426-35.

6. $\mathrm{Wu} C W, \mathrm{Wu} J \mathrm{Y}, \mathrm{Chen} \mathrm{CK}$, et al. Does procalcitonin, C-reactive protein, or interleukin- 6 test have a role in the diagnosis of severe infection in patients with febrile neutropenia? A systematic review and meta-analysis. Support Care Cancer 2015;23:2863-72.

7. Bruno B, Busca A, Vallero $S$, et al. Current use and potential role of procalcitonin in the diagnostic work up and follow up of febrile neutropenia in hematological patients. Expert Rev Hematol 2017;10:543-50.

8. Sbrana A, Torchio M, Comolli G, et al. Use of procalcitonin in clinical oncology: a literature review. New Microbiol 2016;39:174-80.

9. Hemming V, Jakes AD, Shenton G, Phillips B. Prospective cohort study of procalcitonin levels in children with cancer presenting with febrile neutropenia. BMC Pediatr 2017;17:2.
10. Freifeld AG, Bow E), Sepkowitz KA, et al. Clinical practice guideline for the use of antimicrobial agents in neutropenic patients with cancer: 2010 update by the infectious diseases society of america. Clin Infect Dis 2011;52:56-93.

11. Sirinoglu M, Soysal A, Karaaslan A, et al. The diagnostic value of soluble urokinase plasminogen activator receptor compared with C-reactive protein and procalcitonin in children with febrile neutropenia. Pediatr Hematol Oncol 2016;33:200-8.

12. Demirkaya $M$, Tugcu $D$, Akcay $A$, et al. Adrenomedullin-A new marker in febrile neutropenia: comparison with CRP and procalcitonin. Pediatr Hematol Oncol 2015;32:482-9.

13. Hitoglou-Hatzi S, Hatzistilianou M, Gougoustamou D, et al. Serum adenosine deaminase and procalcitonin concentrations in neutropenic febrile children with acute lymphoblastic leukaemia. Clin Exp Med 2005;5:60-5.

14. Kitanovski L, Jazbec J, Hojker S, Gubina M, Derganc M. Diagnostic accuracy of procalcitonin and interleukin- 6 values for predicting bacteremia and clinical sepsis in febrile neutropenix children with cancer. Eur J Clin Microbiology Infect Dis 2006;25:413-5.

15. Hatzistilianou M, Rekleity A, Athanassiou K, DeLutiis MA, Conti $P$, Catriu D. Serial procalcitonin responses in infection of children with secondary immunodeficiency. Clin Invest Med 2007;30:75-85.

16. Robinson JO, Lamoth F, Bally F, Knaup M, Calandra T, Marchetti O. Monitoring procalcitonin in febrile neutropenia: what is its utility for initial diagnosis of infection and reassessment in persistent fever? PLoS One 2011;6:e18886.

17. Stoma I, Karpov I, Uss A, Rummo O, Milanovich N, Iskrov I. Diagnostic value of sepsis biomarkers in hematopoietic stem cell transplant recipients in a condition of high prevalence of gram-negative pathogens. Hematol Oncol Stem Cell Ther 2017; 10:15-21.

18. Fleischhack G, Kambeck I, Cipic D, Hasan C, Bode U. Procalcitonin in paediatric cancer patients: its diagnostic relevance is superior to that of C-reactive protein, interleukin 6, interleukin 8 , soluble interleukin 2 receptor and soluble tumour necrosis factor receptor II. Br J Haematol 2000;111:1093-102.

19. Reitman AJ, Pisk RM, Gates JV $3^{\text {rd }}$, Ozeran JD. Serial procalcitonin levels to detect bacteremia in febrile neutropenia. Clin Pediatr (Phila) 2012;51:1175-83. 